\title{
Is fluoroscopy-guided percutaneous rupture of facet cyst an alternative to surgery? A case report
}

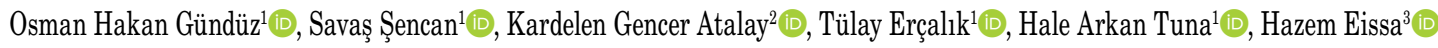 \\ ${ }^{1}$ Pain Medicine Section, Department of Physical Medicine and Rehabilitation, Marmara University Medical School, İstanbul, Turkey \\ ${ }^{2}$ Department of Physical Medicine and Rehabilitation, Marmara University Medical School, İstanbul, Turkey \\ ${ }^{3}$ Department of Pain Medicine, Ochsner Baptist Medical Center, New Orleans, USA
}

Received: October 25, 2017 Accepted: December 22, 2017 Published online: November 04, 2018

\begin{abstract}
Lumbar intraspinal synovial cysts (LISCs) are rare cause of the lumbosacral radicular syndrome. Fluoroscopy-guided percutaneous cyst rupture (PCR) is an option in the treatment. This report introduces long-term symptom relief after fluoroscopy-guided PCR of LISC. A 73-year-old patient presented with low back pain radiating to right leg. L5 radiculopathy was suspected by physical examination; however, an intraspinal cystic mass was visualized through imaging studies. The patient reported immediate pain relief after fluoroscopy-guided PCR, which sustained for one year. In conclusion, fluoroscopy-guided PCR appears to be a safe and effective treatment option and should be considered before surgery in patients with LISCs.
\end{abstract}

Keywords: Fluoroscopy-guided percutaneous cyst rupture; low back pain; lumbar intraspinal synovial cyst.

Lumbar intraspinal synovial cysts (LISCs) are rare intraspinal lesions which may mimic disc herniation with radicular symptoms. These lesions mainly originate from the facet joint capsule. The cyst can be covered with synovium, and the cyst content may be gelatinous or hemorrhagic. They are usually detected by imaging studies, or incidentally during an operation, in patients with low back pain or symptoms of radiculopathy. ${ }^{[1]}$ The incidence rate for patients undergoing lumbar spine surgery ranges from 0.01 to $0.8 \%$. In the cysts evaluated by imaging methods, this ratio is slightly higher, ranging between 0.8 and $2.0 \% .^{[1-3]}$

Lumbar intraspinal synovial cysts mostly originate from the facet joint capsule and are most commonly located at the L4-5 spinal level. ${ }^{[2]}$ The diagnosis is usually made by the presence of a contrast-enhanced capsule on magnetic resonance imaging (MRI). ${ }^{[3]}$ In addition, LISCs may give rise to clinical symptoms including neurogenic claudication, radiculopathy, and low back pain, when it extends to the lumbar spinal canal and compresses the neural elements. Therefore, its symptoms are similar with those of lumbar disc herniation (LDH), although its treatment is entirely different, including fluoroscopy-guided percutaneous cyst rupture (PCR), intra-cystic steroid injection, or surgical treatment options. ${ }^{[4-6]}$ Of note, there is a lack of data on the outcome of percutaneous techniques. Although some authors have suggested that surgical cyst excision is the classical treatment approach, the optimal treatment for LISC remains controversial. In this report, we present a case of LISCs treated with fluoroscopy-guided PCR.

\section{CASE REPORT}

A 73-year-old male patient presented to our clinic with low back pain radiating to the right leg and numbness for a year. His medical and family history were unremarkable. His pain remained unchanged, despite the use of analgesic treatment. On physical examination, the lumbar extension was painful and restricted. The straight leg raise test was bilaterally negative. The right extensor hallucis longus (EHL) muscle strength was $4 / 5$, while the other muscle groups had normal strength. There was hypoesthesia on the 
dorsum of the right foot. The Numeric Rating Score (NRS) for pain was $7 / 10$. In the lumbar MRI, there was a $10 \times 7 \mathrm{~mm}$ ovoid extradural intraspinal cystic formation at the right paramedian location at the level of L4-5, in the neighborhood of the right facet joint. The smoothly contoured lesion appeared $\mathrm{T}_{1}$-hypointense, $\mathrm{T}_{2}$-hyperintense, and it showed a connection with the facet joint; there was also an increase in the joint fluid. Ligamentum flavum hypertrophy was also evident at this level. The lesion was found as a facet joint synovial cyst (Figure 1a, b). The synovial cystinduced mass effect in the L4-5 intervertebral space, thecal sac, and right L5 nerve root compression was also detected. Being unresponsive to conservative treatment, the patient underwent an initial right L5 transforaminal epidural steroid injection (TFESI). There was no reduction in symptoms, neither in the first hour nor the third week. Thus, a fluoroscopyguided percutaneous right L4-5 facet joint cyst rupture was planned. A written informed consent was obtained from the patient.

\section{Procedural technique}

The patient was placed in the prone position and connected to a monitor. Antiseptic measures were taken at the site of intervention. The skin and subcutaneous tissue were anesthetized with a local anesthetic agent. The right L4-L5 facet joint was visualized by fluoroscopy. A 22-gauge 3.5-inch spinal needle was directed at that facet joint. A small volume of contrast was given into the facet joint and the cyst to confirm the localization. Then, more contrast was given and drawn back, and given again under continuous pressure. The cyst rupture was ensured to occur with a reduction of pressure in the injector connected to the needle. Approximately $3 \mathrm{cc}$ seromucinous fluid was aspirated. Re-administration of the contrast material to the location of cyst showed that it was ruptured, with contrast material spreading to the posterior, and then, anterior epidural space (Figure 2). The procedure was well-tolerated. No intraor post-procedural complication was seen. The patient was monitored in the recovery room for two hours and, then, discharged home.

\section{Follow-up}

The patient's leg and low back pain completely vanished at the first hour after the procedure. He was still asymptomatic with a normal physical examination, except for 4/5-muscle strength of right EHL at the third-week and the third-month control visits (NRS 0/10). His physical examination remained
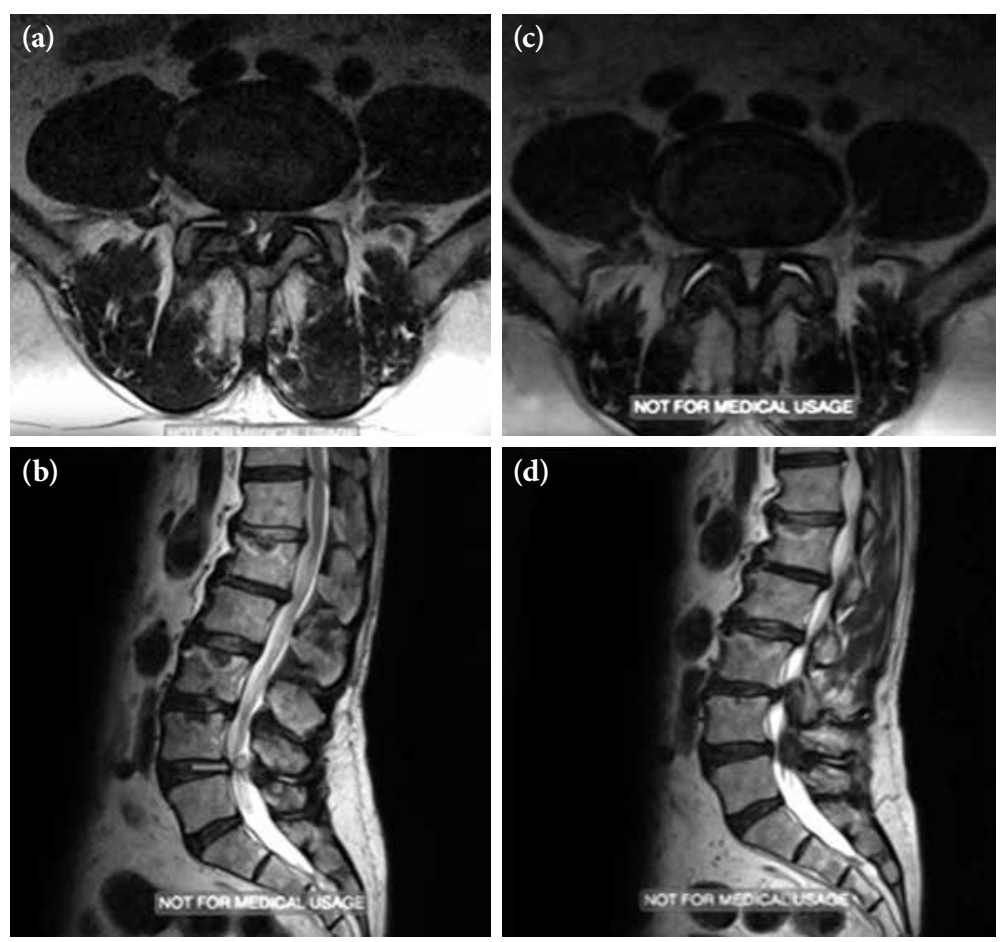

Figure 1. (a, c) L4-5 level axial section, $\mathrm{T}_{2}$ sequence (a) before treatment, (c) after treatment, (b, d) sagittal section $\mathrm{T}_{2}$ sequence, (b) before treatment, (d) after treatment (lumbar intraspinal synovial cyst is seen at the tip of the arrow). 

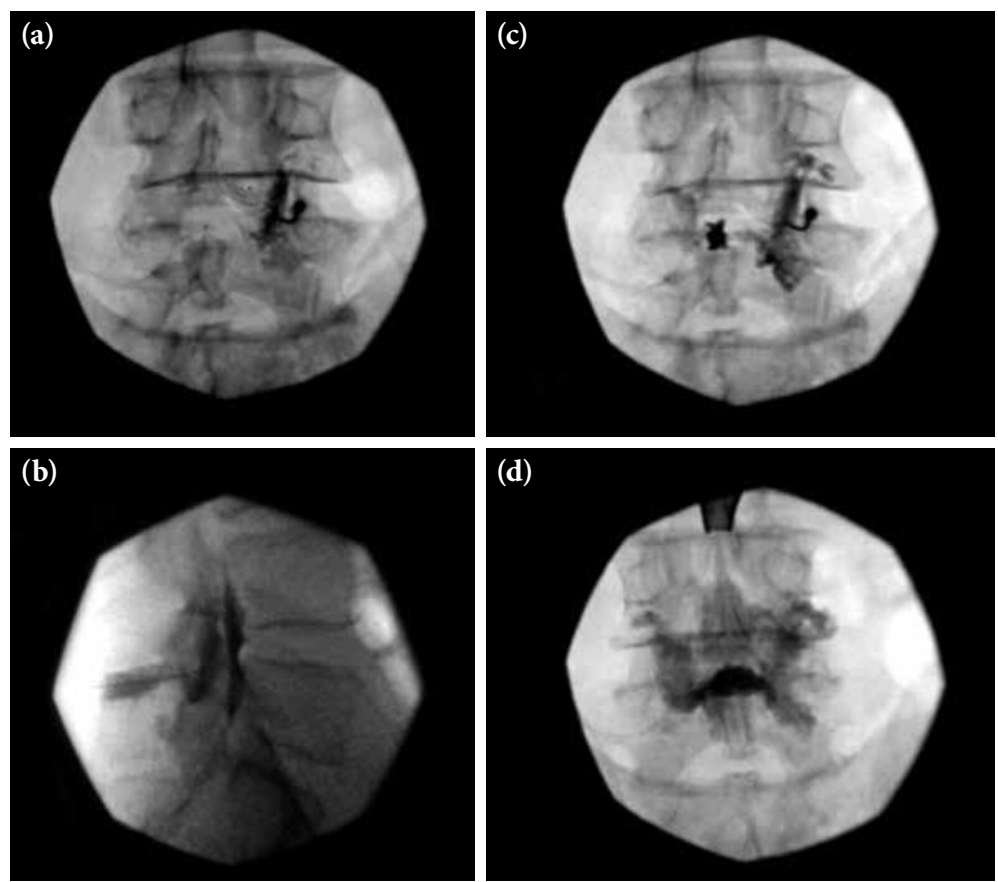

Figure 2. Fluoroscopy-guided, (a) coronal image of L4-5 level facet joint (b) sagittal image of bilateral epidural contrast spread after rupture (c) coronal image of L4-5 level facet joint cyst after rupture (d) coronal image of bilateral epidural contrast spread after rupture (Possible location of the lumbar intraspinal synovial cyst appears at the tip of the arrow).

unchanged at six months and at one year; however, the NRS scores were recorded as $1 / 10$. Control MRI scans were taken at three weeks and at six months. The MRI scan at the first control showed that the cyst entirely disappeared, although a remnant cyst was seen in the second control (Figure 1c, d).

\section{DISCUSSION}

We reported a fluoroscopy-guided PCR procedure in a patient with a facet joint cyst which mimicked LDH presenting with low back pain and radicular symptoms. In the present case, excellent pain relief was achieved throughout the first year. First control MRI at three weeks confirmed the complete elimination of the cyst. Second control MRI at six months showed a remnant cyst formation which did not affect the clinical symptoms of the patient. Therefore, the appearance was not considered as a recurrence.

Our results are also consistent with the results of the complete elimination of pain in 23 of 32 patients with facet joint cyst reported in a case series by Allen et al. ${ }^{[7]}$ However, in the aforementioned study, none of these patients were scheduled for a second control MRI.
The facet cyst rupture procedure was applied after eliciting an incomplete response by right L5 TFESI in our case who presented with a clinical picture of a radiculopathy. Similarly, Melfi and Aprill ${ }^{[8]}$ applied a facet cyst rupture procedure in a 72-year-old female patient with radicular low back pain after an incomplete response to TFESI and facet joint injection. Both reports highlight that facet joint cysts may mimic LDH with radiculopathy symptoms, which may lead to diagnostic delays.

Surgical excision with or without segmental fusion remains the gold standard in the treatment of symptomatic LISCs. However, the literature data suggest that surgical cyst excision may bear some serious risks including dural laceration, cerebrospinal fluid leak, epidural hematoma, spinal instability, discitis, neurological injury, and other problems caused by general anesthesia. ${ }^{[9,10]}$ Thus, our case who tolerated the procedure well without any complication, demonstrates that the facet joint cyst rupture may be an alternative to surgery. We did not observe any recurrence during one-year follow-up period in the present case. Allen et al. ${ }^{[7]}$ reported a recurrence rate of $37.5 \%$ in a case series with a one-year follow-up. ${ }^{[7]}$ 
Even when recurrences develop during follow-up, this procedure can be repeated. This gives superiority to PCR in comparison with surgery. The location of the cyst at the level of L4-5 and the diagnostic accuracy of MRI are other important properties, supporting the literature data. ${ }^{[2,3]}$

Percutaneous cyst rupture can be applied by fluoroscopy or CT guidance. The CT-guided approach may have an advantage thanks to the improved soft tissue contrast and ability to target the cyst directly, on the other hand, this may involve greater radiation exposure. ${ }^{[10,11]}$ Chazen et al. ${ }^{[12]}$ reported successful pain relief with CT-guided PCR on five patients who failed prior fluoroscopic rupture. Either with fluoroscopy or CT guidance, PCR is achieved similarly by forceful pressurization. Nonetheless, there is a need for further studies to examine the superiority or cost-effectiveness of these guidance methods.

In conclusion, our case highlights the rare occurrence of a facet joint cyst that should be considered in the differential diagnosis armamentarium of lumbar radiculopathy. In patients with radicular pain who cannot benefit the available treatments, LISCs should be considered in the differential diagnosis. Fluoroscopy-guided PCR, a safe and efficient minimally invasive treatment technique, should be initially attempted and be taken into consideration as a treatment method alternative to surgery.

\section{Declaration of conflicting interests}

The authors declared no conflicts of interest with respect to the authorship and/or publication of this article.

\section{Funding}

The authors received no financial support for the research and/or authorship of this article.

\section{REFERENCES}

1. Trummer M, Flaschka G, Tillich M, Homann CN, Unger F, Eustacchio S. Diagnosis and surgical management of intraspinal synovial cysts: report of 19 cases. J Neurol Neurosurg Psychiatry 2001;70:74-7.

2. Artico M, Cervoni L, Carloia S, Stevanato G, Mastantuono M, Nucci F. Synovial cysts: clinical and neuroradiological aspects. Acta Neurochir (Wien) 1997;139:176-81.

3. Shah RV, Lutz GE. Lumbar intraspinal synovial cysts: conservative management and review of the world's literature. Spine J 2003;3:479-88.

4. Marichal DA, Bertozzi JC, Rechtine G, Murtagh FR. Case 101: lumbar facet synovial cyst. Radiology 2006;241:618-21.

5. Lyons MK, Atkinson JL, Wharen RE, Deen HG, Zimmerman RS, Lemens SM. Surgical evaluation and management of lumbar synovial cysts: the Mayo Clinic experience. J Neurosurg 2000;93:53-7.

6. Foley BS. Percutaneous rupture of a lumbar synovial facet cyst. Am J Phys Med Rehabil 2009;88:1046.

7. Allen TL, Tatli Y, Lutz GE. Fluoroscopic percutaneous lumbar zygapophyseal joint cyst rupture: a clinical outcome study. Spine J 2009;9:387-95.

8. Melfi RS, AprillCN. Percutaneous puncture of zygapophysial joint synovial cyst with fluoroscopic guidance. Pain Med 2005;6:122-8.

9. Epstein NE. Lumbar synovial cysts: a review of diagnosis, surgical management, and outcome assessment. J Spinal Disord Tech 2004;17:321-5.

10. Shah VN, von Fischer ND, Chin CT, Yuh EL, Amans MR, Dillon WP, et al. Long-Term Effectiveness of Direct CT-Guided Aspiration and Fenestration of Symptomatic Lumbar Facet Synovial Cysts. AJNR Am J Neuroradiol 2018;39:193-8.

11. Bui J, Bogduk N. A systematic review of the effectiveness of CT-guided, lumbar transforaminal injection of steroids. Pain Med 2013;14:1860-5.

12. Chazen JL, Leeman K, Singh JR, Schweitzer A. Percutaneous CT-guided facet joint synovial cyst rupture: Success with refractory cases and technical considerations. Clin Imaging 2018;49:7-11. 